\title{
A Vehicular License Plate Recognition Framework For Skewed Images
}

\author{
M.Y.Arafat, A.S.M.Khairuddin* and R.Paramesran \\ Department of Electrical Engineering, Faculty of Engineering, University of Malaya \\ Kuala Lumpur-50603, MALAYSIA \\ [e-mail: yeasir.ete.ruet09@gmail.com] \\ [e-mail: anissalwa@um.edu.my] \\ [e-mail: ravee@um.edu.my] \\ *Corresponding author: A.S.M Khairuddin
}

Received December 13, 2017; revised April 21, 2018; revised May 22, 2018; accepted June 21, 2018;

published November 30, 2018

\begin{abstract}
Vehicular license plate (LP) recognition system has risen as a significant field of research recently because various explorations are currently being conducted by the researchers to cope with the challenges of LPs which include different illumination and angular situations. This research focused on restricted conditions such as using image of only one vehicle, stationary background, no angular adjustment of the skewed images. A real time vehicular LP recognition scheme is proposed for the skewed images for detection, segmentation and recognition of LP. In this research, a polar co-ordinate transformation procedure is implemented to adjust the skewed vehicular images. Besides that, window scanning procedure is utilized for the candidate localization that is based on the texture characteristics of the image. Then, connected component analysis (CCA) is implemented to the binary image for character segmentation where the pixels get connected in an eight-point neighbourhood process. Finally, optical character recognition is implemented for the recognition of the characters. For measuring the performance of this experiment, 300 skewed images of different illumination conditions with various tilt angles have been tested. The results show that proposed method able to achieve accuracy of $96.3 \%$ in localizing, $95.4 \%$ in segmenting and $94.2 \%$ in recognizing the LPs with an average localization time of $0.52 \mathrm{~s}$.
\end{abstract}

Keywords: License plates (LP), intelligent transport systems (ITS), character recognition, skewed images. 


\section{Introduction}

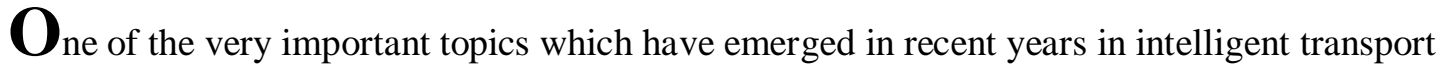
systems (ITS) is the vehicular license plate (LP) recognition system. Vehicular license plate recognition (VLPR) system gets utilized for detecting vehicles. It provides a reference as well for further vehicle activity analysis and tracking. VLPR system has become a core methodology because of its wide range of traffic applications along with security ranging from parking automation to vehicle surveillance, electrical tollgate management, restricted area security control, road traffic monitoring, analysis of vehicle activity, tracking for safety and calculating the traffic volume $[1,2]$.

VLPR systems should operate properly or attain real-time performance with relatively less processing time for fulfilling the requirements of ITS, where 'real-time' indicates the operational process throughout the image of identifying every desired single object with relatively faster processing. VLPR system is generally composed of basic three stages which are the license plate detection (LPD), the character segmentation and the character recognition whilst LPD has emerged as the most important stage in the VLPR system since the scheme's accuracy gets influenced by it [3]. Moreover, because of the parameter diversities involved in the vehicle images, LPD has become the most crucial stage among these three steps [1, 4].

For recognizing the vehicular license plates many approaches have been proposed by many researchers but the promising scenarios like tracking the number plates from speeding vehicles, skewed vehicle images, blurry and lower resolution images have been addressed in very few researches. Because of low contrast images, crowded background, skewed images and weak edge information the inefficiency in localizing the vehicle number plate area still exists despite the procedures proposed in previous works.

In most of the existing VLPR systems the number plate text had been assumed to be lying in a plane and in that cases the angles with respect to the optical axis of the sensor are generally normal [5]. But in case of skewed images the angular adjustment is the precursor for proper recognition performance.

This paper focused on all the three basic steps which are the license plate detection (LPD), character segmentation and recognition [6]. Moreover, in this study a polar co-ordinate transformation based procedure has been proposed for the proper adjustment of the skewed vehicular images. In this study a framework has been proposed which consists of five stages (pre-processing, skew correction, candidate localization, character segmentation and recognition) for overcoming the challenges mentioned above.

This paper has been methodized as follows. Firstly, a related work on the existing VLPR system has been discussed in Section 2. In Section 3, the proposed approach has been explained. The experimental results have been provided in Section 4. Finally, the paper has been concluded in Section 5 with remarks.

\section{Related Work}

Many approaches have been proposed by many researchers for recognizing the vehicular license plates but most of them focused on detection part only and in many cases single vehicular image had been utilized. VLPR system has become a core methodology because of the fast progress in urban areas and the rapid traffic congestion. Different attributes such as character existence, license plate region boundary, texture, combined features have been 
considered in detecting the vehicular number plates. In order to locate the desired number plate regions in image data or videos from the previous procedures could be categorized into three parts i.e. gray-level processing, binary image processing, and color processing [7]. Generally vehicular plates holding license information possess the shape of quadrangles along with particular aspect ratio. This boundary information based procedures have been widely implemented in $[3,8,9]$ for locating this quadrangles or rectangular shapes. Texture is known as the color changing between the characters and the number plate background. One of the egregious tools for this texture analysis is considered the Gabor filtering [10]. But the drawback of this filtering is the computational complexity which has been reported as comparatively high [4]. Mathematical morphology, vertical gradient have been used in [11]. A frequency domain procedure integrated with multi-resolution analysis (MRA) in wavelet has been utilized in [12] where the featured had been extracted with respect to the abruptly changed intensity values.

CCA (Connected Component Analysis) integrated technique has been implemented as one of the significant methodologies for the processing of the binary images [2, 13]. In [14] an algorithm has been implemented for tracking out the connected objects through utilizing the contour detection. The objects that get selected to be the desired candidate within these connected objects possess the identical geometrical attributes as that of the vehicular plate. The character segmentation stage gets influenced if there is skewed image. The Hough transform technique has been utilized in [13] for the corrective adjustment of the rotation problem of the vehicular plate images. A hybrid binarization technique has been utilized after the horizontal scan line analysis on the license plate images.

Another procedure integrated with Radon transform has been implemented in [5] for correcting the rotational problem in accordance with horizontal perspective. In [14] the binary perimeter image has been utilized firstly by applying Bernsen binarization and then Radon transform has been utilized for tilt angle correction. The color combination between the characters and the vehicular number plates is a unique feature which is also utilized for tracking the desired plate region. A neural network implemented procedure for the classification of the each pixels color information has been utilized in [15] where before this classification the RGB constituents had been converted into HLS (Hue, Lightness, and Saturation) model.

For character segmentation various attributes i.e. vertical and horizontal projection attributes [7, 16], pixel connectivity [17, 18], character contour feature [19], mathematical morphology [20] and characters prior knowledge [13, 21] have been utilized. The segmentation accuracy has a great influence for proper recognition rate since majority of the recognition errors in VLPR framework occur because of the segmentation errors. Complications such as non-uniform brightness, unpredictable shadows, angular skew of the LP, physical damage need to be handled properly.

Character recognition stage bears a very important role in VLPR framework through identifying the desired number of the LP. For Character recognition different attributes i.e. pattern or template matching algorithm [7], implementing extracted attributes [22], deploying classifiers such as Artificial Neural Networks (ANN) [2] or statistical classifiers i.e. Hidden Markov Model (HMM) [23], fuzzy SVM (Support Vector Machine) [24] have been utilized.

Implementing pattern or template matching attributes are straightforward and relatively simpler technique. Implementing extracted attributes enable faster recognition, capable of extracting the salient attributes, robust in distinguishing the characters in case of distortion. For implementing classifiers the recognition system requires additional processing time for 
training the network and possess processing complexity but attains higher recognition efficiency in case of huge amount of data.

\section{Proposed Method}

The proposed Vehicular License Plate Recognition (VLPR) framework aims at overcoming the drawbacks of the existing methods. Proposed VLPR approach consists of basic four phases: pre-processing, skew correction, candidate localization (region extraction \& VLP detection), and character segmentation \& recognition. Fig. 1 depicts the phases of the proposed VLPR method sequentially.

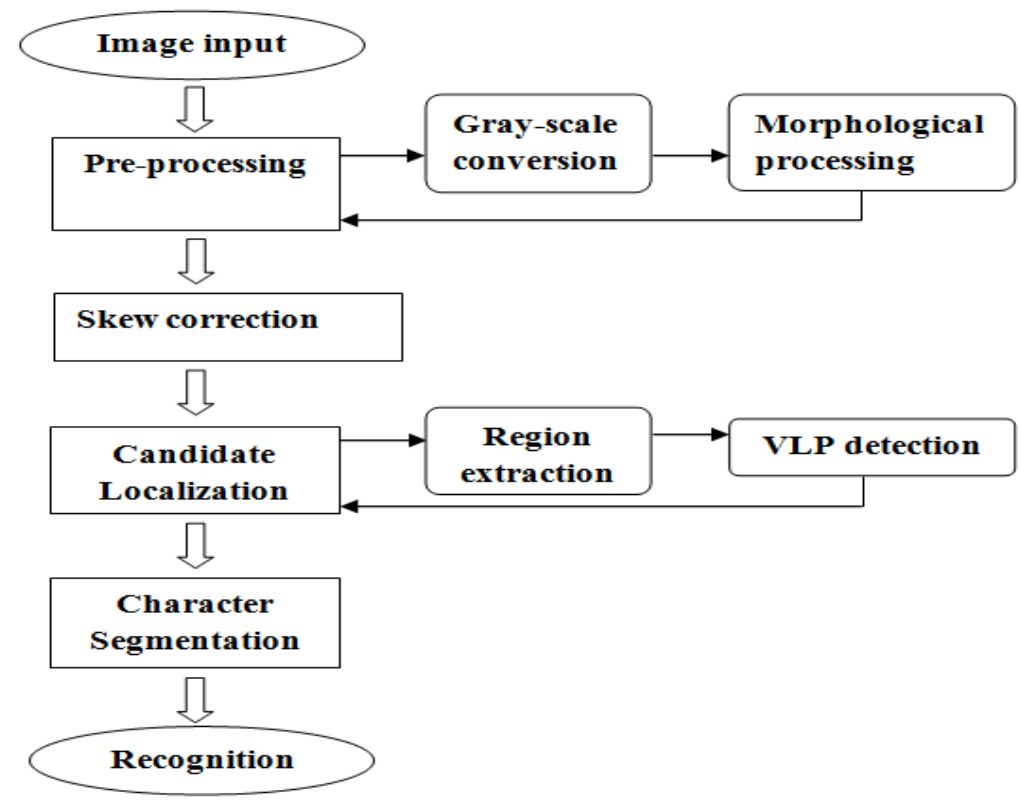

Fig. 1. Flowchart of the proposed approach sequentially.

\subsection{Pre-processing}

Pre-processing is the preliminary phase in the digital image processing which improves the quality of the image data for both proper visual perception and computational processing. Pre-processing enhances the image data by removing both background noise, unwanted data, image reflections and normalising the intensities of the individual image particles. A major reason for the failure of vehicle license plate detection is the low quality of the vehicle image data [4]. In this work, the Pre-processing stage comprised of two major sub-processes such as conversion of the RGB color image into gray-scale and morphological processing which improves the contrast of the image data at such locations where there might be a possibility of holding the vehicle license candidate.

\subsubsection{Gray-scale conversion}

The process of producing gray-scaled images from color (RGB) images is known as gray-scaling. The threshold of the image data is calculated in this procedure. When this value is smaller than the threshold value then to find out the proper gray-scale value, it is necessary 
to recalculate the image data. The intention of thresholding is for splitting the point of concern from the background. When an image was loaded into matlab then, a matrix $M$ (3 dimensional) of size ( $\mathrm{ZxYxX}$ ) with $\mathrm{Z}$ and $\mathrm{Y}$ being the number of pixels in $\mathrm{z}$ - and $\mathrm{y}$-direction was obtained. Generally, this matrix is for the RGB images. The values for all the three colors are identical when it is a grayscale image; they generally range between 0 and 255 . The threshold is applied to find out the proper gray-scale value. The threshold value is determined by the different intensity levels. The image data quality gets enhanced for further smooth computational processing by this gray-scaling procedure. Here,

Threshold = t;

values below $=(\mathrm{M}<\mathrm{t})$;

values above $=(\mathrm{M}>=\mathrm{t})$;

The values below then get set to black $M$ (values below) $=0$; and the values above then get set to white $\mathrm{M}$ (values above) $=255$.

This technique has an important role by providing the necessary contrast of the image data. This helps in differentiating between the separate levels of intensities of the background and the object for smooth computational processing.

From the RGB (color) value (24-bit) of each corresponding pixel $(x, y)$; the Red, Green and Blue components are being separated and the (8-bit) gray level (converted) value is calculated by using this [25] following formula:

$$
S(x, y)=1 / 10\{3 \times R(x, y)+6 \times G(x, y)+1 \times B(x, y)\}
$$

Here, $\mathrm{R}(x, y), \mathrm{G}(x, y)$ and $\mathrm{B}(x, y)$ represents the spectrum of Red, Green and Blue components respectively and $\mathrm{S}(x, y)$ indicates the converted gray-scaled image of the input RGB image which has been depicted in Fig. 2(a).

\subsubsection{Morphological Processing}

This image processing operation involves in morphological transformation by combining two sets through vector addition of the set elements [26]. Here, input image data gets improvised through this operation by: joining the broken-lines, enhancing the brightness, sharpening the edges of objects and filling holes of the input image data.

Assuming that, $E$ and $F$ being the sets in Z-space $\left(P^{Z}\right)$ including elements $e$ and $f$, respectively, $e=\left(e_{1}, e_{2}, \ldots, e_{Z}\right)$ and $f=\left(f_{1}, f_{2}, \ldots, f_{Z}\right)$. As a result the subsets of $P^{Z}$ is $e$ and $f$. Hence, $E \oplus F$ indicates the dilation operation of $E$ by $F$ and is defined by using [26] the equation as follows:

$E \oplus F=\left\{j \in P^{Z} \mid j=e+f\right.$ for some $e \in E$ and $\left.f \in F\right\}$

Here, the dilation operation gets along by both the close \& open arithmetic operations [11]. For close: Set $F$ close aggregate set $E$ as follows:

$$
E \bullet F=(E \oplus F) \Theta F
$$

For open: Set Fopen aggregate set Eas follows:

$$
E \circ F=(E \Theta F) \oplus F
$$

There are several important tasks both for close and open arithmetic operations. The close arithmetic is involved in filling the smaller holes of the objects, sharpening the edges, smoothing the boundary of the objects, connecting the neighbourhood objects whereas open arithmetic is involved in eliminating relatively smaller objects, smoothing of the boundary of the relatively large objects, separating the objects at the fine places. 
(a)

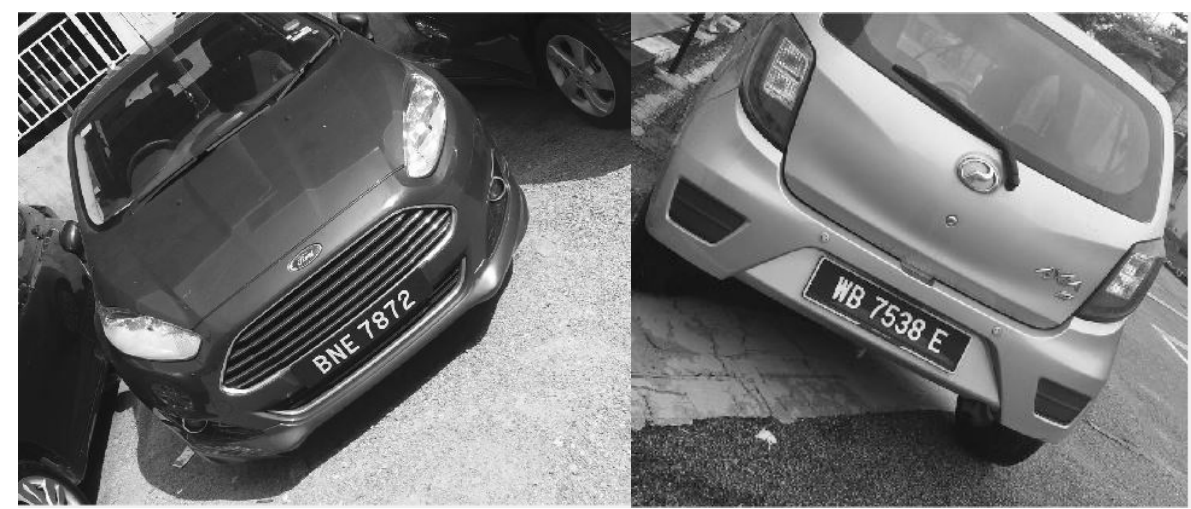

(b)

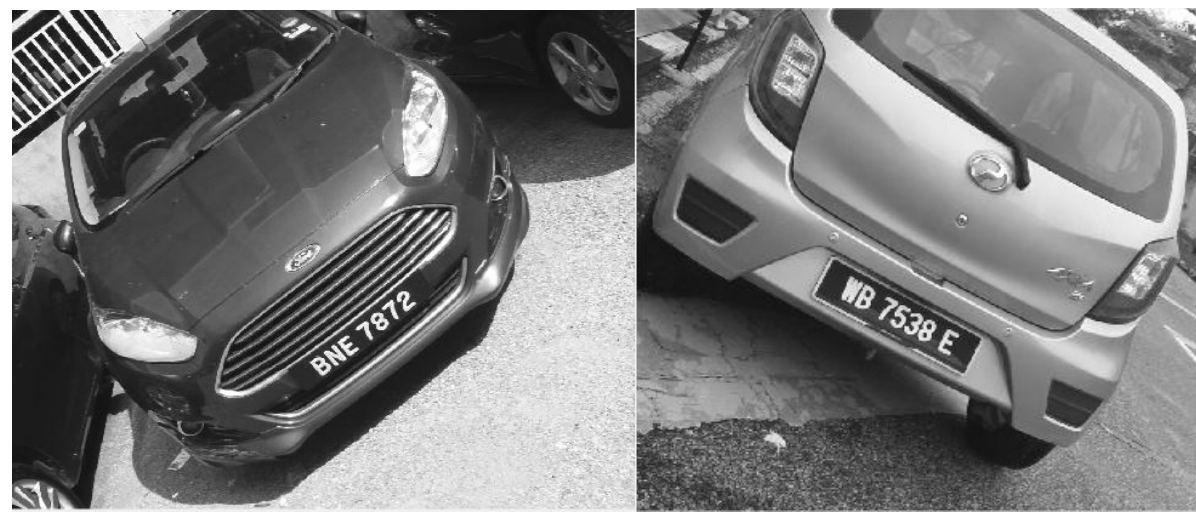

Fig. 2. Vehicular images after: (a) gray-scale conversion (b) morphological processing

In this research, Malaysian vehicle images have been utilized. Here, close arithmetic operation has been performed in this research resulting in smoothing the boundary of the objects. After morphological processing, the edges get sharper. As a result the gray value difference between the two neighbouring pixels gets enhanced specially at the edges of the object. Fig. 2(b) depicts the vehicular images after morphological processing.

\subsection{Skew correction}

Regarding the vehicle license plate images which are inclined, the characters including the image, also become inevitably inclined. As a result, the tilting adjustment of the characters becomes necessary precursor in order to get the characters in identical horizontally adjusted position. Not only for improving the accuracy of the character recognition step but also to facilitate character segmentation this tilting adjustment is very advantageous. This adjustment process works on the basis of average-height of image pixels. In general, for several images, which includes various characters, from both left and right parts of the character the pixel height needs to be horizontally placed at a nearer position. The image entity gets inclined in case of the relatively high fluctuations and then corrective adjustment is required [27].

For the tilt adjustment, the pixels average heights from both left and right parts of the image need to be enumerated firstly and afterwards the slope is determined because when the pixel 
height of the character from the right and left sides shows relatively high fluctuations, the image existence shows to be inclined. The image gets reorganised in accordance with the image inclined slope by utilizing polar co-ordinate transformation procedure by proper revolving. This includes in the pixel mapping of new image to the old image for getting this Euclidean entity under the projective distortion.

The procedure is described briefly in three steps as follows:

$>$ Progressive in accordance with scanning the image through column from the left part first and afterwards counts aggregates the height; then the image pixels average height from the left part is enumerated.

$>$ Repeat step 1 replacing the left part by the right part.

$>$ The slope is now calculated in accordance with the pixels average heights from both left and right parts of the image.

In general, images consisting of numerous characters have possibilities of possessing the pixel height of the character from the right and left sides at a close horizontal position. In case of the relatively high fluctuations, the image existence shows to be inclined. For this tilt adjustment, the pixels average heights from both left and right parts of the image need to be enumerated firstly and afterwards the slope is determined. The image is readjusted then in accordance with the skewed slope of the image by utilizing polar co-ordinate transformation procedure by proper revolving. The co-ordinates $\left(a_{x}, a_{y}\right)$ of the image pixel are set $\alpha$ degrees rotation clockwise yielding the co-ordinate $\left(b_{x}, b_{y}\right)$. Before rotation, the pixels polar co-ordinate can be expressed by the following equations:

$$
\begin{aligned}
& a_{x}=m \cos (\beta) \\
& a_{y}=m \sin (\beta)
\end{aligned}
$$

Here, $m$ is the slope and $\beta$ is the angle between $x$-axis and the co-ordinates $\left(a_{x}, a_{y}\right)$.

This relationship is illustrated at the Fig. 3 as follows:

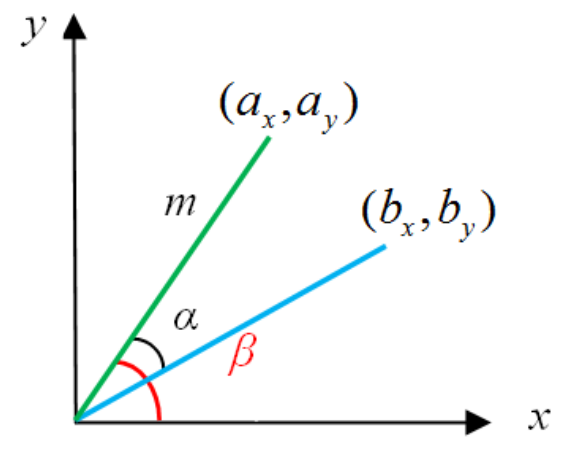

Fig. 3. Pixel revolving diagram.

After revolving $\alpha$ :

$$
\begin{aligned}
b_{x} & =m \cos (\beta-\alpha) \\
& =m\{\cos (\beta) \cos (\alpha)+\sin (\beta) \sin (\alpha)\} \\
& =m \cos (\beta) \cos (\alpha)+m \sin (\beta) \sin (\alpha)
\end{aligned}
$$




$$
\begin{aligned}
& =a_{x} \cos (\alpha)+a_{y} \sin (\alpha) \\
b_{y} & =m \sin (\beta-\alpha) \\
& =m\{\sin (\beta) \cos (\alpha)-\cos (\beta) \sin (\alpha)\} \\
& =m \sin (\beta) \cos (\alpha)-m \cos (\beta) \sin (\alpha) \\
& =a_{y} \cos (\alpha)-a_{x} \sin (\alpha) \\
& =-a_{x} \sin (\alpha)+a_{y} \cos (\alpha)
\end{aligned}
$$

The inclined image gets reorganised in accordance with the inclined slope by utilizing polar co-ordinate transformation procedure by proper revolving. This includes in the pixel mapping of new image to the old image for getting this projective distortion.

This experiment focuses on the skewed vehicle images and tilt adjustment has been performed on the entire vehicle image as the image gets reorganised in accordance with the tilted slope. The adjustment of the tilted slope takes place where the pixel heights fluctuations are relatively high. The car detection process is performed after tilt adjustment.

Equation (7) \& (8) can be expressed as matrix as follows:

$$
\left[\begin{array}{c}
b_{x} \\
b_{y} \\
1
\end{array}\right]=\left[\begin{array}{ccc}
\cos \alpha & \sin \alpha & 0 \\
-\sin \alpha & \cos \alpha & 0 \\
0 & 0 & 1
\end{array}\right]\left[\begin{array}{c}
a_{x} \\
a_{y} \\
1
\end{array}\right]
$$

In accordance with the above formula, the introduced matrix expression is as follows:

$$
\left[\begin{array}{c}
a_{x} \\
a_{y} \\
1
\end{array}\right]=\left[\begin{array}{ccc}
\cos \alpha & -\sin \alpha & 0 \\
\sin \alpha & \cos \alpha & 0 \\
0 & 0 & 1
\end{array}\right]\left[\begin{array}{c}
b_{x} \\
b_{y} \\
1
\end{array}\right]
$$

In conformity with equation (9), after revolving the image, for every point, the corresponding image can be obtained as Fig. 4 .

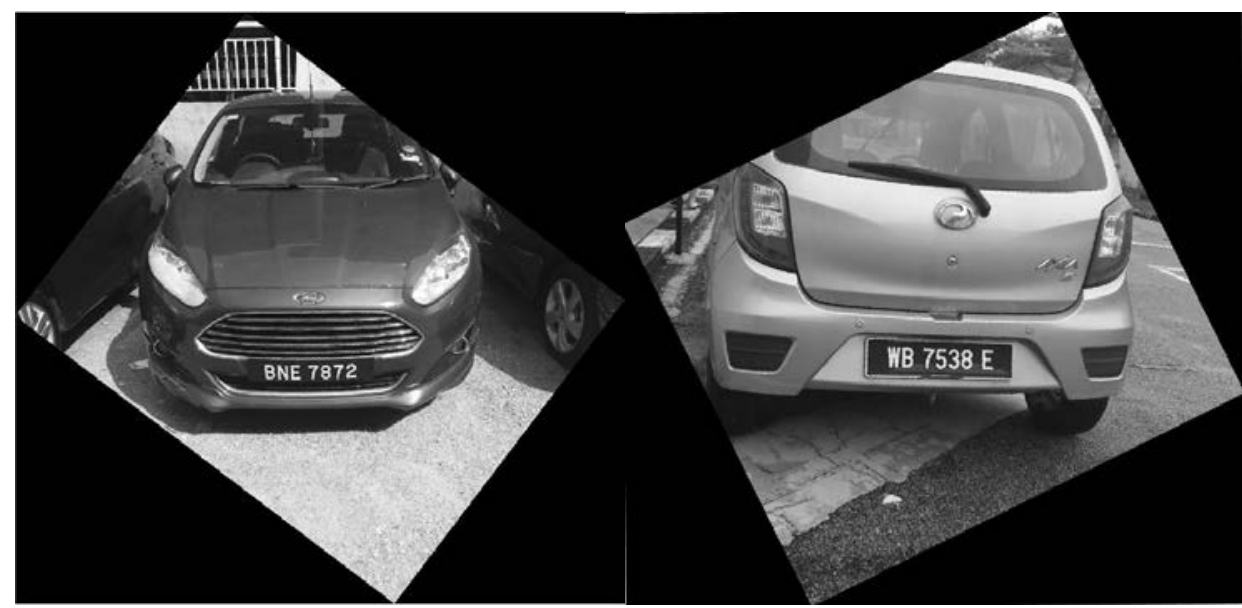

Fig. 4. Vehicle images after skew correction. 


\subsection{Candidate Localization}

In most cases, candidate localization has become one of the significant precursors for the VLPR recognition framework [7]. This stage has been comprised of two basic sub-processes i.e. region extraction and VLP (Vehicular License Plate) detection for the proper recognition framework as follows:

\subsubsection{Region Extraction}

Here, in this region extraction stage, the candidates probable set of quadrangles that contain the highest possibilities of possessing the LP are investigated. This candidature region extraction has been methodized on the basis of the texture characteristics of the images by considering the fact of frequent transient differences or rapid spatial variation of image in the probable candidate region that are much more than other areas of the vehicle image. Here, for the extraction of the probable set of quadrangles that possess the larger possibilities of attaining the LP, window scanning procedure has been utilized.

The procedure is explained here briefly with the basic stages:

Stage 1: Initially for an input image $g(a, b)$ with size of $(x \times y)$, the aggregate of the transient differences for each of the windows are being enumerated.

$$
\begin{aligned}
& \text { For } q=1: y-1 \\
& \quad \text { For } p=1: x-X_{W}-1 \\
& S_{T}=\sum_{i=p}^{p+X_{W}} g(i, q)-g(i+1, q)
\end{aligned}
$$

Here, the window starts with the coordinate $(p, q)$ where $X_{W}$ denotes the width of the window.

Stage 2: Secondly, the aggregate of the transient differences for each of the windows are stored in the variable, $S_{T}$ as the window traverses.

$$
\text { If } \left.\left(S_{T}\right\rangle T\right)
$$

Set $R^{p}=1$

Else set $R^{p}=0$

Here, $T$ is used for the threshold value. The threshold $T$ is utilized for adjusting the aggregate of the transient differences for each of the windows. This threshold is automatically adjusted by the algorithm for selecting the consecutive rows to be summed up for each of the windows.

Stage 3: Thirdly, the consecutive rows are summed up, labelled $R$ to one line after line. Then the aggregated amount of the consecutive rows is stored into $L$.

$$
\begin{gathered}
\text { For } p=1: y-1 \\
\text { If } R^{p}=1,(p=r, r+1, r+2, \ldots, n) \\
\text { Set } L=\sum_{p=r}^{n} R^{p}
\end{gathered}
$$

Stage 4: $\quad$ If $\left(L>T_{T}\right)$

$$
\text { Set } A_{\text {end }}^{z}=p \text {, }
$$




$$
A_{\text {initial }}^{z}=p-L \quad(z=1,2,3, \ldots . .)
$$

Where $T_{T}$ indicates an adequate threshold value, $z$ denotes the probable regions for the candidate. These regions are being extracted as the probable candidate in accordance with the arrays $A_{\text {initial }}$ and $A_{\text {end }}$.

There are some candidate-regions which have been achieved after applying the stages above.

The threshold value in this stage $T_{T}$ is used after the consecutive rows get aggregated. This threshold is selected as half of the total consecutive rows that are summed up. The probable regions are that areas which possess the highest transient differences or spatial variations. Above this threshold value $T_{T}$, the candidate regions are extracted as probable region possessing the LP. These probable regions are shown in Fig. 5 as follows:

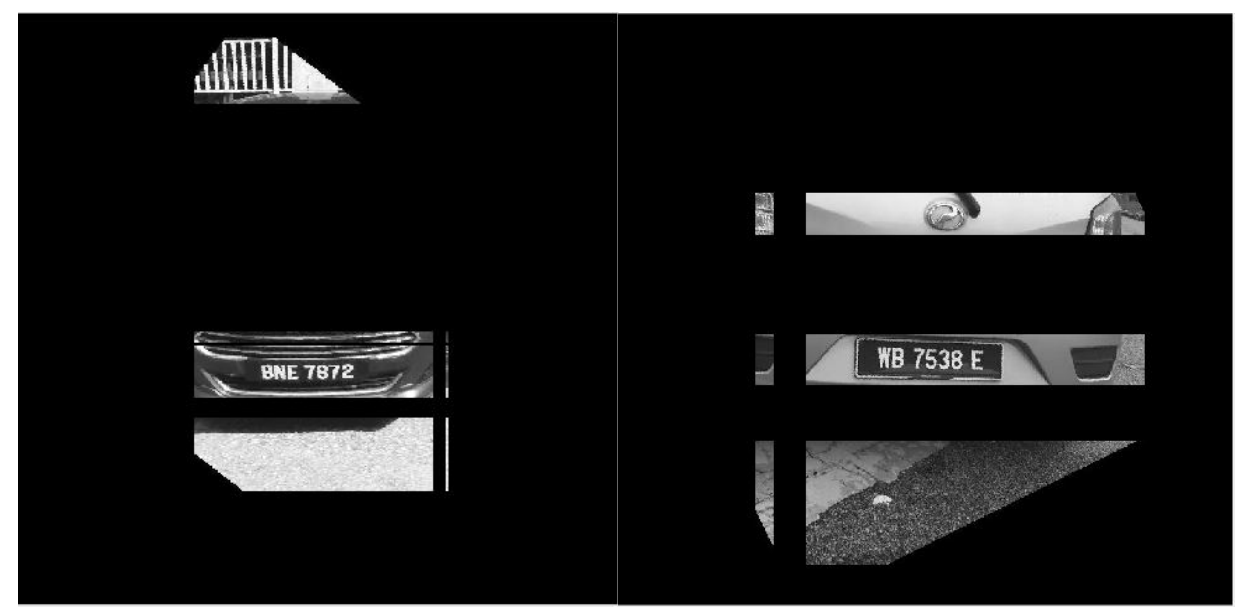

Fig. 5. Extracted candidate plate images

Then finally the exact location of the vehicular LP gets detected (subsection 3.3.2) after using the dynamic threshold which is the highest value of the transient differences. The corresponding regions having transient difference values lower than the threshold are then separated and the exact locus gets detected.

\subsubsection{VLP Detection}

The final stage of determining the correct location of vehicular license plate (VLP) is the VLP detection. The output from the region extraction step attains the highest possibility of containing the exact locus of the vehicular LP. Here, the final output of the region extraction step contains some other portions along with this VLP of the input image data. Among these parts of the probable candidate region, the part possessing the highest transient differences, comprises the highest possibility of containing the exact locus of the vehicular LP. In order to find out the region attaining the highest transient differences, a dynamic threshold value is to be implemented and the transient differences of the linear windows for the probable candidates are being processed by this value of threshold. The outcome from this process yields the portion comprising the maximum probability of possessing the exact locus of the vehicular license plate (VLP) finally. Fig. 6 depicts the detected vehicular LP here as follows: 


\section{BNE 7872 WB $7538 \mathrm{E}$}

Fig. 6. Detected vehicular LP

\subsection{Character segmentation \& recognition}

This character segmentation stage performs a very important role in this vehicular license plate recognition framework for the proper recognition of the vehicular LP. Here, in order to perform character segmentation properly on the plate image, it needs to undergo through binarization process by changing the grey-scale values of the image into binary. Therefore the pixels at the background get suppressed and the pixels that are for interest get highlighted. Then the connected component analysis (CCA) is implemented [28] which is an image processing application in which the image is scanned first and the corresponding pixels are then labelled into components in accordance with the pixel connectivity. For this case identical pixel intensity-values are shared by all of the pixels in a specified connected component. These pixels then get connected among themselves in some way (either get four-connected or get eight-connected). Each of the pixels is then labelled with a value in accordance with the component with which it got assigned after all groups had been determined. CCA works on the grey-level or the binary images with different forms of connectivity. Here, for this experiment CCA has been implemented on the binary image in order to search for an eight-connected component situation.

Let, $\delta v$ be an eight-point neighbourhood process where $c(v)$ denotes the neighbour set which is connected to point $v$. The set $c(v)$ ought to acquire the following properties for all $v$ and $l$ :

$c(v) \subset \delta v$

$l \in c(v) \Leftrightarrow v \in C(l)$

The domain $D \subset V$ will get connected under $c(y)$ if there for all $y, l \in D$, exists an order of $N$ pixels: $v_{1}, v_{2}, \ldots, v_{N}$ such that: $v_{1} \in C(v), v_{2} \in C\left(v_{1}\right), \ldots, v_{N} \in C\left(v_{N-1}\right), l \in C\left(v_{N}\right)$.

The connected components get extracted by the following algorithm:

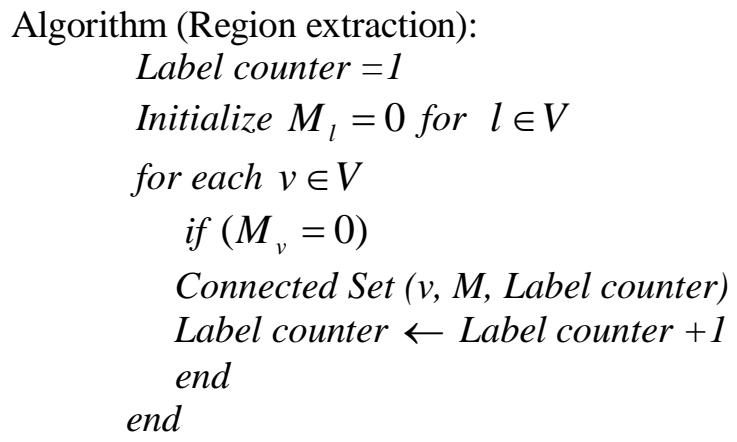

Then the blob (binary large object) [29] assessment technique is implemented which belongs the strong architecture for determining the contactless and closed regions in the binary image.

The procedure is described briefly in five steps as follows:

Step 1: First of all the label counter which is initiated to one is created. Then the binary image gets scanned.

Step 2: For the selected region-criterion, every pixel is checked for the eight-connectivity. When a neighbour matches due to the criterion, the pixel is then assigned to that region. 
Step 3: For the case of multiple neighbours that fit result in all the numbers are of the equivalent region and the pixels are assigned to their region.

Step 4: For case of no neighbours fitting the criteria, the region counter value is assigned and then the region counter is increased by one. Afterwards for assigning the same region value to all the equivalent regions, the image is scanned again.

Step 5: The procedure continues in the image as soon as there left no unlabeled pixels. Here, the extracted characters are depicted in Fig. 7 in the green boxes as follows:

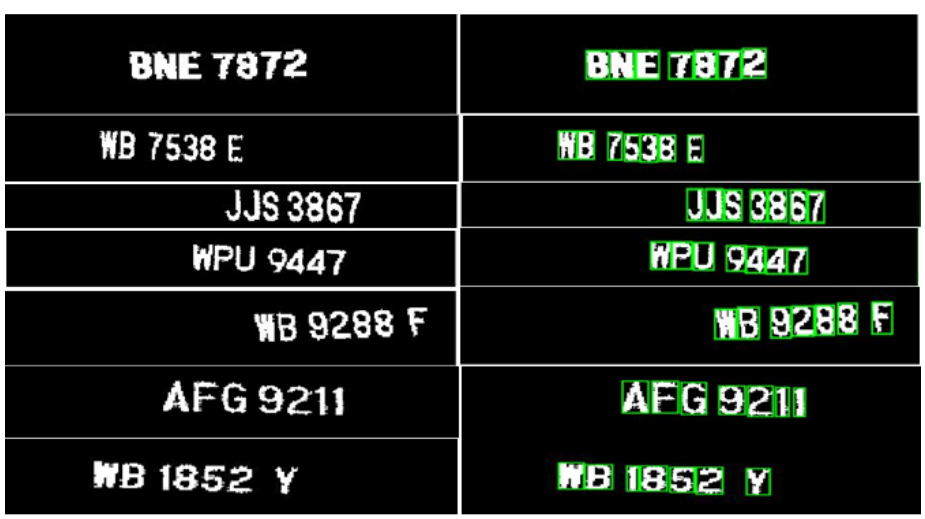

Fig. 7. Character extracted plate image (Blob assessment output)

After that the cell array is created in order to store the segmented characters individually.

The indexed characters are then returned according to the corresponding element numbers and then saved in the cell array.

The procedure is described briefly in three steps as follows:

Step 1: Loop over through every blob that already has been detected.

Step 2: The blob of pixels is then to be extracted in order to acquire each of the characters.

Step 3: Placing the characters into the cell array by defining a cell array: cell $(\mathrm{x}, \mathrm{y})$ which is the empty matrices of $x$ by y cell array where $y$ is according to the number of elements in the array. Fig. 8 depicts all the segmented characters of the vehicular license plate individually according to the cell arrays.

The final part of this Vehicular License Plate Recognition (VLPR) framework is the character recognition. After all of these procedures in character segmentation stage are carried out through the image, the characters become much more proficient for the optical character recognition (OCR) system in order to perfect recognition in this Vehicular License Plate Recognition (VLPR) framework. Template matching technique has been implemented in Matlab for the optical character recognition.

\section{Experimental Results}

The proposed algorithm has been evaluated on an Intel ${ }^{\circledR}$ Core $^{\mathrm{TM}}$ i3 CPU including $4.00 \mathrm{~GB}$ RAM with Windows 8 at clock speed $3.70 \mathrm{GHz}$ and 64-bit operating system for this experiment. For measuring the performance of this experiment 300 skewed images of different illumination conditions with various tilt angles have been tested. 


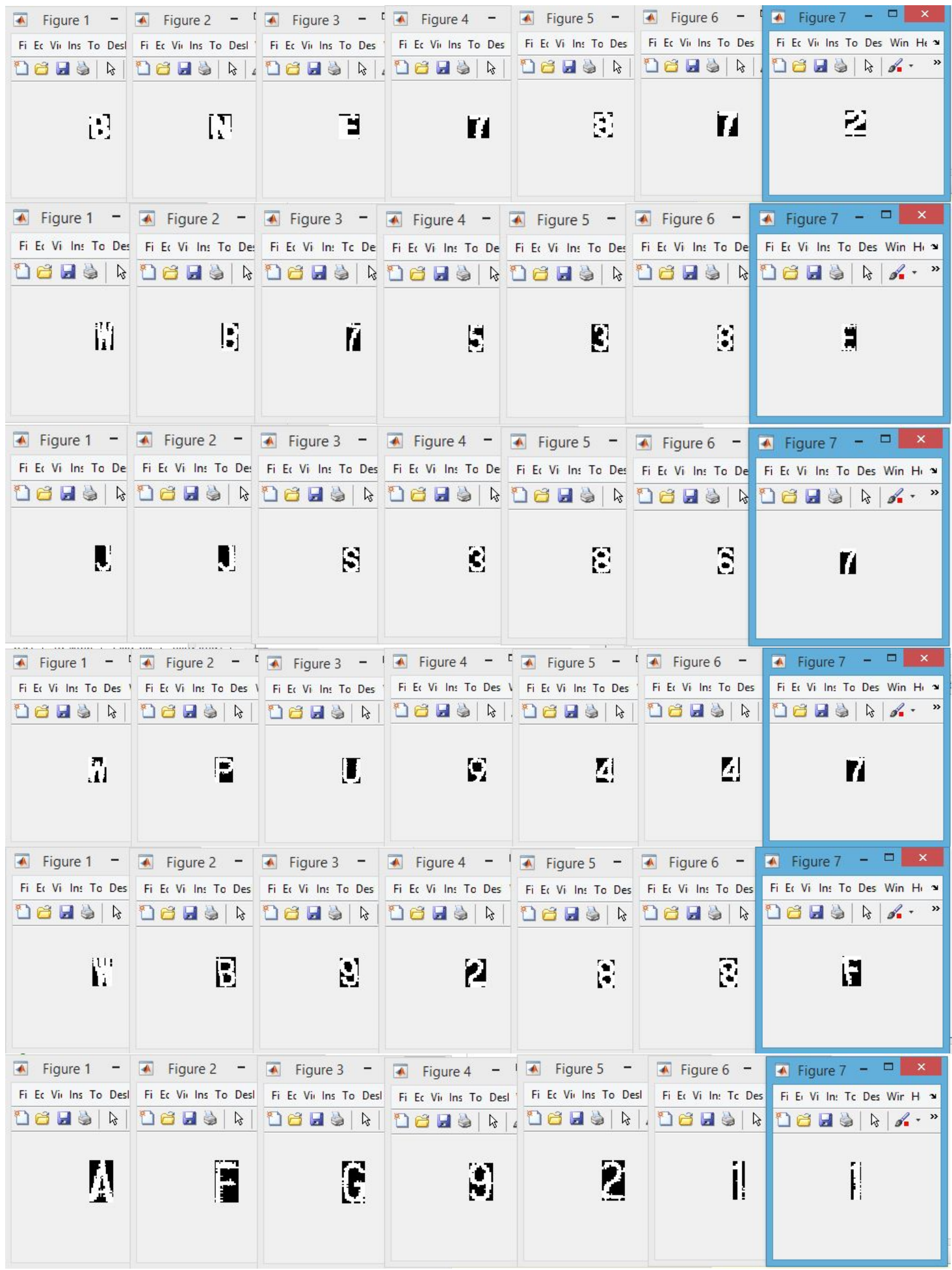




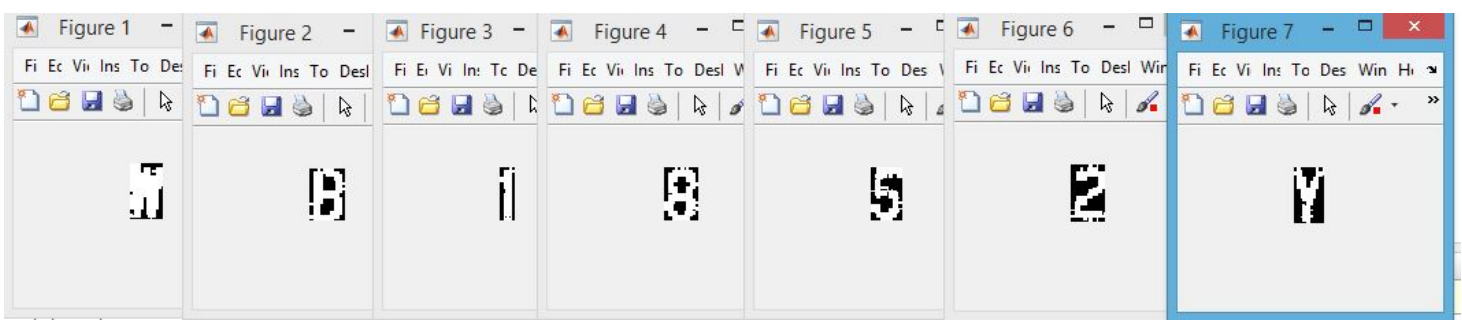

Fig. 8. Segmented characters of the Vehicular LP individually

The LP localization, character segmentation and recognition results have been summarized in the Table 1 as follows:

Table 1. Results for LP localization, character segmentation and recognition systems

\begin{tabular}{|c|c|c|}
\hline LP localization stage & Quantity & Percentage \\
\hline \hline The number of total tested vehicle images & 300 & 100 \\
\hline Correctly detected LP images & 289 & 96.3 \\
\hline Images with unsuccessful detection & 11 & 3.7 \\
\hline Character segmentation stage & 2100 & 100 \\
\hline Total character numbers & 2004 & 95.4 \\
\hline Successful character segmentation & 96 & 4.6 \\
\hline Unsuccessful character segmentation & & 100 \\
\hline Character recognition stage & 2100 & 94.2 \\
\hline Total character numbers & 1978 & 5.8 \\
\hline Successful character recognition & 122 & \\
\hline Unsuccessful character recognition & & \\
\hline
\end{tabular}

This procedure has achieved a noteworthy performance. The rate of LP localization has achieved an accuracy of $96.3 \%$, character segmentation attained a success rate of $95.4 \%$ and the character recognition achieved an accuracy of $94.2 \%$ which satisfies the procedure to be helpful for the real time applications. The recognized characters of the vehicular license plate have been depicted in Fig. 9 individually after the pattern matching as follows: 

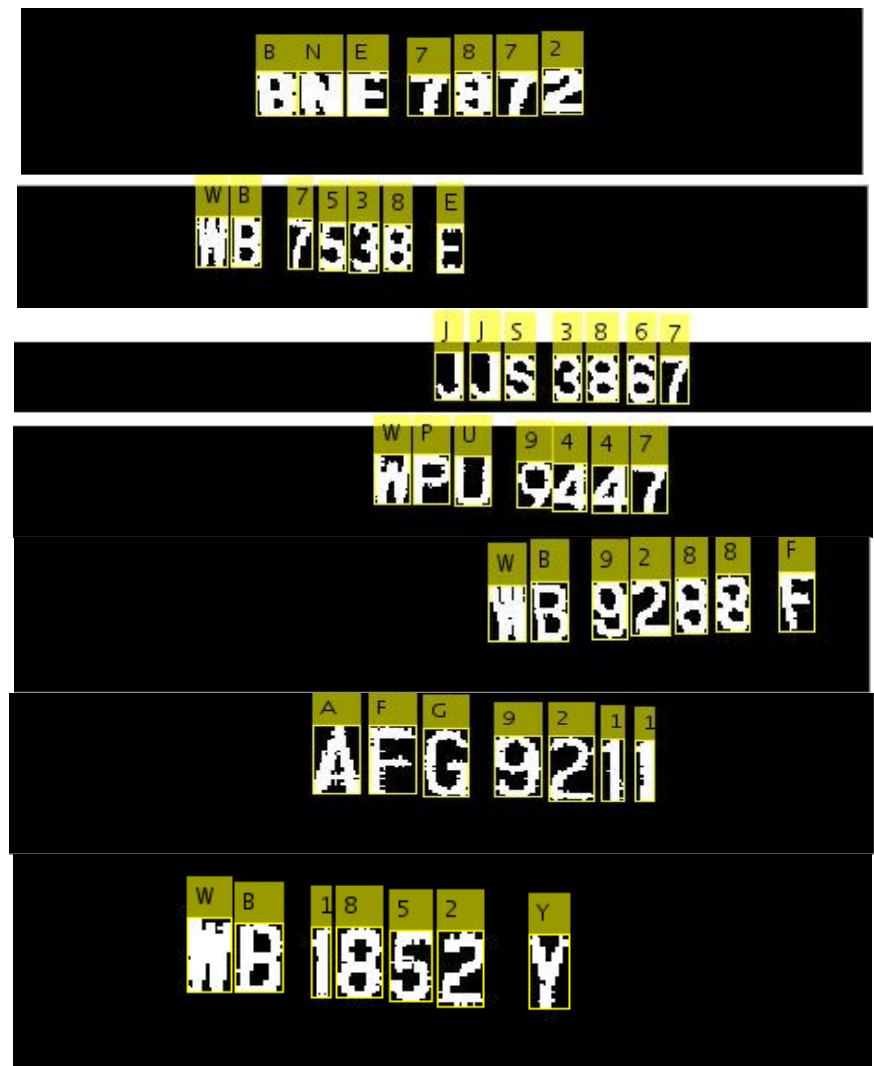

Fig. 9. Character recognition of the vehicular LP

The performance of the proposed system has been compared with respect to some existing procedures in the Table 2 as follows:

Table 2. Performance comparison with respect to some existing systems

\begin{tabular}{|c|c|c|c|c|}
\hline Reference & $\begin{array}{c}\text { LP localization } \\
\text { rate }\end{array}$ & $\begin{array}{c}\text { Character } \\
\text { segmentation rate }\end{array}$ & $\begin{array}{c}\text { Character } \\
\text { recognition rate }\end{array}$ & $\begin{array}{c}\text { Skew } \\
\text { correction }\end{array}$ \\
\hline \hline$[30]$ & $82.5 \%$ & - & - & - \\
\hline$[5]$ & $98 \%$ & - & - & yes \\
\hline$[31]$ & - & $84.5 \%$ & - & - \\
\hline$[32]$ & $96.8 \%$ & $91.1 \%$ & $87.5 \%$ & - \\
\hline$[1]$ & $93.86 \%$ & - & - & - \\
\hline$[33]$ & $97.27 \%$ & - & - & yes \\
\hline This method & $96.3 \%$ & $95.4 \%$ & $94.2 \%$ & \\
\hline
\end{tabular}

Some unsuccessful image samples have been depicted in Fig. 10 as follows: 
(a)

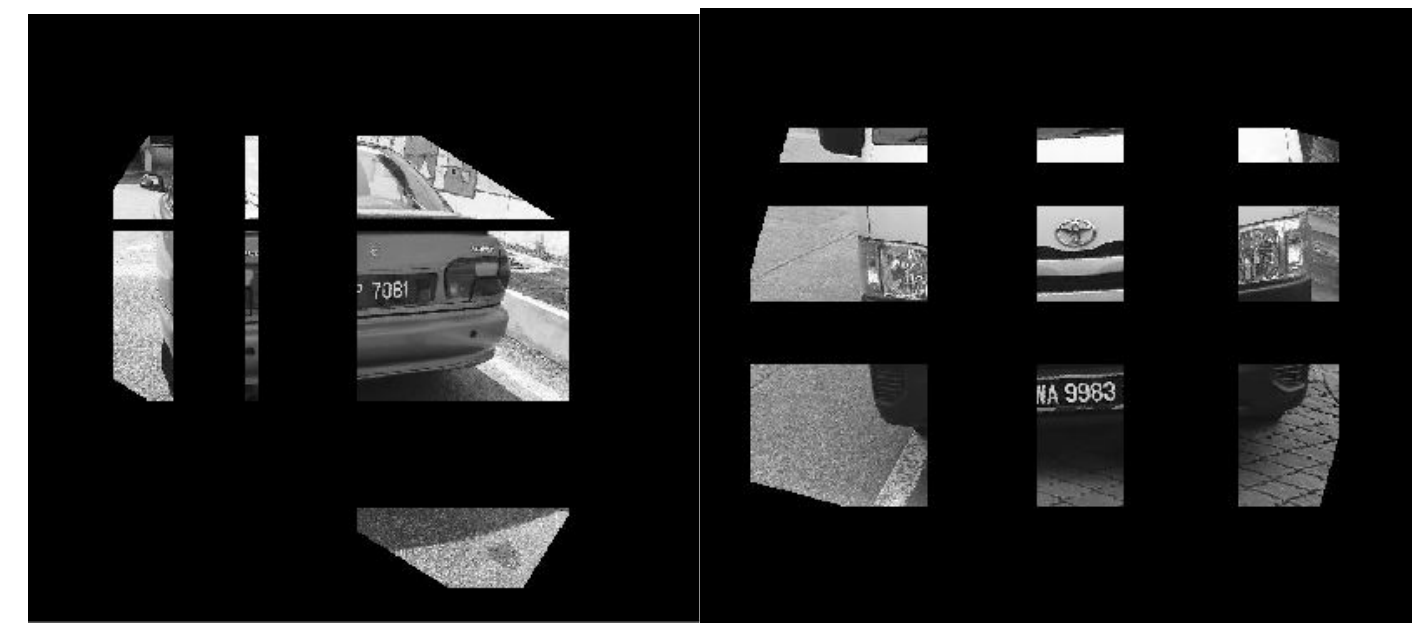

\section{MT15266:}

\section{BLE AIOTO}

(b)

\section{AEE FIOS}

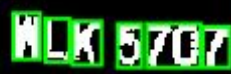
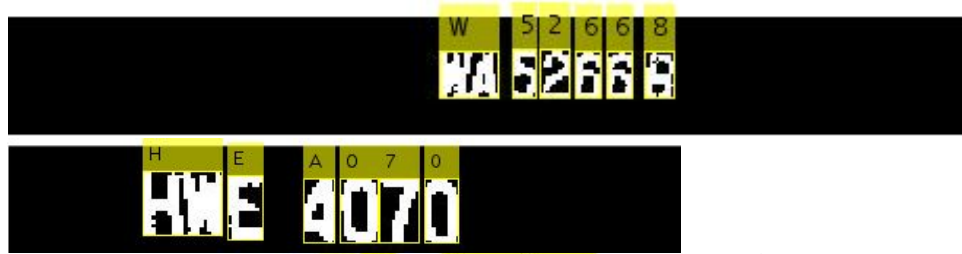

(c)
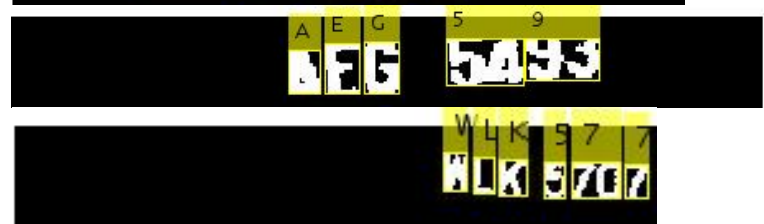

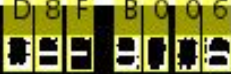

Fig. 10. Unsuccessful samples of: (a) localization (b) character segmentation (c) character recognition 


\section{Conclusion}

In this research, a skew correction technique where the image gets reorganised in accordance with the image inclined slope by utilizing polar co-ordinate transformation procedure has been presented here along with a VLPR recognition framework. Experiments were done in 3 stages: LP localization stage, character segmentation stage and character recognition stage. The results tabulated that unsuccessful detected images is $3.7 \%$, unsuccessful character segmentation is $4.6 \%$ while unsuccessful character recognition is $5.8 \%$ for LP localization stage, character segmentation stage and character recognition stage respectively. Hence, the proposed system possesses a noteworthy performance which proves the approach to be helpful for the real time applications. For future study, license plate recognition from speeding vehicles, blurry and darker images will be investigated. Besides that, recognition of LP for images with multivehicles will be explored.

\section{References}

[1] M. R. Asif, Q. Chun, S. Hussain, and M. S. Fareed, "Multiple licence plate detection for Chinese vehicles in dense traffic scenarios," IET Intelligent Transport Systems, vol. 10, pp. 535-544, 2016. Article (CrossRef Link).

[2] İ. Türkyılmaz and K. Kaçan, "License Plate Recognition System Using Artificial Neural Networks," ETRI Journal, vol. 39, pp. 163-172, 2017. Article (CrossRef Link).

[3] B. Hongliang and L. Changping, "A hybrid license plate extraction method based on edge statistics and morphology," in Proc. of Pattern Recognition, 2004. ICPR 2004. Proceedings of the 17th International Conference on, pp. 831-834, 2004. Article (CrossRef Link).

[4] V. Abolghasemi and A. Ahmadyfard, "An edge-based color-aided method for license plate detection," Image and Vision Computing, vol. 27, pp. 1134-1142, 2009.

Article (CrossRef Link).

[5] H. Rajput, T. Som, and S. Kar, "Using Radon Transform to Recognize Skewed Images of Vehicular License Plates," Computer, vol. 49, pp. 59-65, 2016. Article (CrossRef Link).

[6] M.-R. Choi and S.-S. Lee, "An image enhancement Method for extracting multi-license plate region," KSII Transactions on Internet \& Information Systems, vol. 11, 2017.

Article (CrossRef Link).

[7] H. Rajput, T. Som, and S. Kar, "An Automated Vehicle License Plate Recognition System," Computer, vol. 8, pp. 56-61, 2015. Article (CrossRef Link).

[8] P. Tarabek, "Fast license plate detection based on edge density and integral edge image," in Proc. of Applied Machine Intelligence and Informatics (SAMI), 2012 IEEE 10th International Symposium on, pp. 37-40, 2012. Article (CrossRef Link).

[9] R. Chen and Y. Luo, "An improved license plate location method based on edge detection," Physics Procedia, vol. 24, pp. 1350-1356, 2012. Article (CrossRef Link).

[10] H. Caner, H. S. Gecim, and A. Z. Alkar, "Efficient embedded neural-network-based license plate recognition system," IEEE Transactions on Vehicular Technology, vol. 57, pp. 2675-2683, 2008. Article (CrossRef Link).

[11] F. Yang and Z. Ma, "Vehicle license plate location based on histogramming and mathematical morphology," in Proc. of Fourth IEEE Workshop on Automatic Identification Advanced Technologies (AutoID'05), pp. 89-94, 2005. Article (CrossRef Link).

[12] V. Laxmi, D. Mohanta, and B. Karan, "Comparison of different wavelets for automatic identification of vehicle license plate," IET intelligent transport systems, vol. 5, pp. 231-240, 2011. Article (CrossRef Link). 
[13] J.-M. Guo and Y.-F. Liu, "License plate localization and character segmentation with feedback self-learning and hybrid binarization techniques," IEEE Transactions on Vehicular Technology, vol. 57, pp. 1417-1424, 2008. Article (CrossRef Link).

[14] S. Azam and M. M. Islam, "Automatic license plate detection in hazardous condition," Journal of Visual Communication and Image Representation, vol. 36, pp. 172-186, 2016.

Article (CrossRef Link).

[15] E. R. Lee, P. K. Kim, and H. J. Kim, "Automatic recognition of a car license plate using color image processing," in Proc. of Image Processing, 1994. Proceedings. ICIP-94., IEEE International Conference, pp. 301-305, 1994. Article (CrossRef Link).

[16] L. Zheng, X. He, B. Samali, and L. T. Yang, "Accuracy enhancement for license plate recognition," in Proc. of Computer and Information Technology (CIT), 2010 IEEE 10th International Conference on, pp. 511-516, 2010. Article (CrossRef Link).

[17] S.-L. Chang, L.-S. Chen, Y.-C. Chung, and S.-W. Chen, "Automatic license plate recognition," IEEE transactions on intelligent transportation systems, vol. 5, pp. 42-53, 2004.

Article (CrossRef Link).

[18] R. Panahi and I. Gholampour, "Accurate detection and recognition of dirty vehicle plate numbers for high-speed applications," IEEE Transactions on Intelligent Transportation Systems, vol. 18, pp. 767-779, 2017. Article (CrossRef Link).

[19] A. Capar and M. Gokmen, "Concurrent segmentation and recognition with shape-driven fast marching methods," in Proc. of Pattern Recognition, 2006. ICPR 2006. 18th International Conference on, pp. 155-158, 2006. Article (CrossRef Link).

[20] S. Nomura, K. Yamanaka, O. Katai, H. Kawakami, and T. Shiose, "A novel adaptive morphological approach for degraded character image segmentation," Pattern Recognition, vol. 38, pp. 1961-1975, 2005. Article (CrossRef Link).

[21] Q. Gao, X. Wang, and G. Xie, "License plate recognition based on prior knowledge," in Proc. of Automation and Logistics, 2007 IEEE International Conference on, pp. 2964-2968, 2007. Article (CrossRef Link).

[22] F. Aghdasi and H. Ndungo, "Automatic licence plate recognition system," in Proc. of AFRICON, 2004. 7th AFRICON Conference in Africa, pp. 45-50, 2004.

Article (CrossRef Link).

[23] D. Llorens, A. Marzal, V. Palazón, and J. M. Vilar, "Car license plates extraction and recognition based on connected components analysis and HMM decoding," in Proc. of Iberian Conference on Pattern Recognition and Image Analysis, pp. 571-578, 2005.

Article (CrossRef Link).

[24] H. Samma, C. P. Lim, J. M. Saleh, and S. A. Suandi, "A memetic-based fuzzy support vector machine model and its application to license plate recognition," Memetic computing, vol. 8, pp. 235-251, 2016. Article (CrossRef Link).

[25] M. K. Sarker, S. Yoon, and D. S. Park, "A Fast and Robust License Plate Detection Algorithm Based on Two-stage Cascade AdaBoost," KSII Transactions on Internet \& Information Systems, vol. 8, 2014. Article (CrossRef Link).

[26] R. M. Haralick, S. R. Sternberg, and X. Zhuang, "Image analysis using mathematical morphology," IEEE transactions on pattern analysis and machine intelligence, pp. 532-550, 1987. Article (CrossRef Link).

[27] W. Zhai, T. Gao, Y. Hu, and Y. Tian, "Research and Implementation of License Plate Character Segmentation Based on Tilt Correction," Information and Automation, pp. 92-97, 2011. Article (CrossRef Link).

[28] W. Zhai, T. Gao, Y. Hu, and Y. Tian, "Research and Implementation of License Plate Character Segmentation Based on Tilt Correction," Information and Automation, pp. 92-97, 2011. Article (CrossRef Link). 
[29] H. E. Kocer and K. K. Cevik, "Artificial neural networks based vehicle license plate recognition," Procedia Computer Science, vol. 3, pp. 1033-1037, 2011.

Article (CrossRef Link).

[30] K. Deb, H.-U. Chae, and K.-H. Jo, "Vehicle License Plate Detection Method Based on Sliding Concentric Windows and Histogram," JCP, vol. 4, pp. 771-777, 2009.

Article (CrossRef Link).

[31] D.-J. Kang, "Dynamic programming-based method for extraction of license plate numbers of speeding vehicles on the highway," International Journal of Automotive Technology, vol. 10, pp. 205-210, 2009. Article (CrossRef Link).

[32] M.-L. Wang, Y.-H. Liu, B.-Y. Liao, Y.-S. Lin, and M.-F. Horng, "A vehicle license plate recognition system based on spatial/frequency domain filtering and neural networks," in Proc. of International Conference on Computational Collective Intelligence, pp. 63-70, 2010. Article (CrossRef Link).

[33] R. Al-Hmouz and K. Aboura, "License plate localization using a statistical analysis of Discrete Fourier Transform signal," Computers \& Electrical Engineering, vol. 40, pp. 982-992, 2014. Article (CrossRef Link).

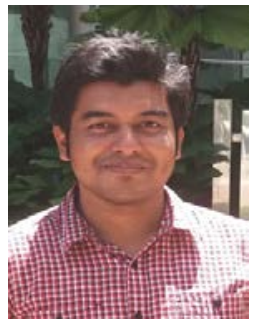

M.Y.Arafat received the Bachelor ofElectronics \& Telecommunication Engineering (ETE) from the Rajshahi University of Engineering \& Technology (RUET), Rajshahi, Bangladesh in 2014.Currently he is a research assistant and M.Sc candidate at the department of electrical engineering, University of Malaya, Kuala Lumpur, Malaysia. His research interests include image processing, intelligent transportation systems (ITSs), pattern recognition and computer vision.

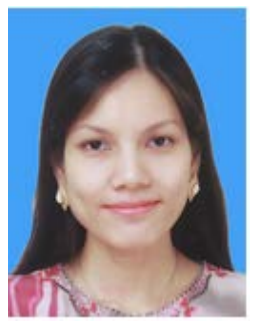

A.S.M Khairuddin (corresponding author) received the Bachelor of Electrical Engineering from the UniversitiTenagaNasional, Malaysia, in 2008, and Master of Computer Engineering from Royal Melbourne Institute of Technology, Australia in 2010 and the Ph.D. degree in electrical engineering from UniversitiTeknologi Malaysia, in 2014. She is currently working as a senior lecturer at Department of Electrical Engineering, Faculty of Engineering, University of Malaya, Malaysia. Her research interests include expert system, pattern recognition and texture analysis.

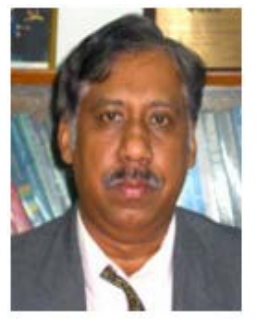

R.Paramesran received the B.Sc degree in 1984 and the M.Sc degree in 1985 in Electrical Engineering from South Dakota State University, Brookings, South Dakota, USA. He was a systems designer with Daktronics, U.S.A. He received his Ph.D degree in 1994 from the University of Tokushima, Japan. He is currently working as a professor at Department of Electrical Engineering, Faculty of Engineering, University of Malaya, Malaysia.His research interests include formulation of new image descriptors for image analysis,image and video analysis, analysis of EEG signals, fast computation of orthogonal moments. He holds a patent for predicting the blood glucose levels using non-parametric model. Currently he is a senior member at IEEE, a member of the Signal Processing Society as well. 